\title{
Induction of sperm acrosome reaction by perivitelline membrane glycoprotein ZP1 in Japanese quail (Coturnix japonica)
}

\author{
Tomohiro Sasanami ${ }^{1}$, Takeomi Murata ${ }^{1}$, Mamoru Ohtsuki ${ }^{1,2}$, Kayoko Matsushima ${ }^{1,2}$, \\ Gen Hiyama ${ }^{3}$, Norio Kansaku ${ }^{3}$ and Makoto Mori ${ }^{1}$ \\ ${ }^{1}$ Department of Applied Biological Chemistry, Faculty of Agriculture, Shizuoka University, 836 Ohya, Shizuoka \\ 422-8529, Japan, ${ }^{2}$ The United Graduate School of Agricultural Science, Gifu University, Gifu 501-1193, Japan and \\ ${ }^{3}$ Laboratory of Animal Genetics and Breeding, Azabu University, Sagamihara 229-8501, Japan \\ Correspondence should be addressed to T Sasanami; Email: atsasan@agr.shizuoka.ac.jp
}

\begin{abstract}
The extracellular matrix surrounding avian oocytes, called the perivitelline membrane (PL), consists of at least two major glycoproteins, ZP3 and ZP1. Our previous study using Japanese quail had demonstrated that the PL obtained from the preovulatory follicles was incubated in vitro with spermatozoa, and perforations were observed. This result indicated that the PL might contain a constituent that possesses activity to initiate the acrosome reaction (AR) in quail. In order to elaborate upon our previous findings, we evaluated the effects of ZP3 and ZP1 on the induction of sperm AR in Japanese quail. Ejaculated sperm were incubated with or without the purified PL glycoprotein, and their acrosome status was determined based on the presence or absence of the acrosome. Treatment of spermatozoa with increasing doses of the purified monomeric ZP1 led to a concentration-dependent stimulation of AR. The purified dimeric ZP1 had similar effect. Moreover, we found that the ZP1-induced AR was significantly blocked by the digestion of the PL protein with PNGaseF. In contrast, the addition of purified ZP3 failed to induce AR at any doses tested. These results indicate that $\mathrm{N}$-linked glycans on ZP1 play an important role in triggering the AR in Japanese quail.

Reproduction (2007) 133 41-49
\end{abstract}

\section{Introduction}

Fertilization is known as the process of combining two germ cells, egg and sperm, and is the consequence of precisely ordered multiple steps, including sperm-egg binding, induction of the acrosome reaction (AR) by sperm, and fusion of sperm and egg. It is well known that the zona pellucida (ZP), which is an extracellular matrix surrounding mammalian oocytes, plays an important role during fertilization because it possesses receptor molecules responsible for relatively species-specific sperm-egg binding, induces the sperm $A R$, as well as participates in the prevention of polyspermy achieved by its structural changes (Florman \& Ducibella 2006). In mammals, the ZP family of glycoproteins has been identified as playing a key role in these events (Wassarman 1999). The components of this matrix include three glycoproteins (i.e. ZP1, ZP2, and ZP3, which are also known as ZPB1, ZPA, and ZPC respectively, according to Spargo \& Hope (2003) and Smith et al. (2005)) in most mammalian species (Harris et al. 1994) and four glycoproteins in humans (ZP1, ZP2, ZP3, and ZP4 (ZPB2); Lefievre et al. 2004).

In avian species, the inner layer of the vitelline membrane (also referred to as the perivitelline membrane (PL)), which is the egg envelope homologous to the $\mathrm{ZP}$ in mammals, is observed in follicles between granulosa cells and the ovum before ovulation (Wyburn et al. 1965). In birds, fertilization occurs within the infundibulum portion of the oviduct, and only the PL encloses the oocyte at the time of fertilization. At least two glycoproteins have been identified as constituents of the avian PL; ZP1 (ZPB1) and ZP3 (ZPC) in Japanese quail (Pan et al. 2001, Sasanami et al. 2003) and ZP1 (ZPB1), ZPB (ZPB2), ZP2 (ZPA), ZP3 (ZPC), and ZPD in chicken (Waclawek et al. 1998, Bausek et al. 2000, Okumura et al. 2004, Smith et al. 2005). We have previously cloned the cDNA encoding ZP3 (GenBank Accession no. AB012606) and ZP1 (GenBank Accession no. AB061520) of the quail PL.

Koyanagi et al. (1988) have demonstrated in the chicken that when the PL obtained from ovulated ova is 
incubated with sperm in vitro, fragmentation of the $\mathrm{PL}$ occurred. Moreover, Howarth (1990) reported that solubilized PL contains one or more components that behave in a manner analogous to sperm receptor in mammalian species, since pretreatment of spermatozoa with solubilized PL eliminates their binding to and fragmentation of the PL in the chicken. In our previous study using Japanese quail, we observed perforations in $\mathrm{PL}$ obtained from preovulatory follicles after it was incubated in vitro with spermatozoa (Kuroki \& Mori 1997). Similarly, Win et al. (2006) also confirmed the formation of holes in PL after the incubation with sperm in Japanese quail. These results indicate that the PL might contain a constituent that initiates the AR in quail. The aim of the present study was to determine which components in the PL possess the activity for the induction of sperm AR in Japanese quail. In order to achieve this goal, we established a method to discriminate acrosome-reacted from acrosome-intact sperm. By means of this method, we show that ZP1 of the PL possesses activity to induce the AR in Japanese quail. We also provide evidence that the $N$-linked oligosaccharide attached to the ZP1 plays an important role in triggering the AR.

\section{Materials and Methods}

\section{Animals and tissue preparation}

Male and female Japanese quail, Coturnix japonica, 15-30 weeks of age (Tokai-Yuki, Toyohashi, Japan), were caged individually under a lighting schedule of $14 \mathrm{~h}$ light (0500-1900 h) and $10 \mathrm{~h}$ darkness, and the animals were provided with tap water and a commercial diet (TokaiKigyo, Toyohashi, Japan) ad libitum. The female animals were decapitated and the largest preovulatory follicles were dissected. The granulosa layer from the largest preovulatory follicles was isolated as a sheet of granulosa cells sandwiched between the PL and the basal laminae, as previously described (Gilbert et al. 1977). A proctodeal gland secretion was obtained manually from male quail as meringue-like foam. This form was then centrifuged at $10000 \mathrm{~g}$ for $10 \mathrm{~min}$ at $4{ }^{\circ} \mathrm{C}$. After centrifugation, supernatants were collected and were stored as proctodeal gland secretion at $-80^{\circ} \mathrm{C}$ until use. All experimental procedures for the use and the care of animals in the present study were approved by the Animal Care Committee of the Faculty of Agriculture, Shizuoka University.

\section{Semen collection and preparation}

Ejaculated semen was obtained from male quail prior to mating according to the procedure of Kuroki \& Mori (1997). Semen obtained from two to three males was suspended in Hanks' balanced salt solution (HBSS) containing $1.25 \mathrm{mmol} / \mathrm{l}$ of $\mathrm{CaCl}_{2}$ and $0.1 \%(\mathrm{v} / \mathrm{v})$ of proctodeal gland secretion. We decided to include $\mathrm{CaCl}_{2}$ in the sperm extender because it is reported that the induction of $\mathrm{AR}$ by homogenized $\mathrm{PL}$ was almost negligible in the absence of $\mathrm{Ca}^{2+}$ (Ashizawa et al. 2006). We added proctodeal gland secretion into the incubation mixture since it inhibits an agglutination of quail spermatozoa. The concentrations of spermatozoa were measured with a hemocytometer and the sperm viabilities were determined using LIVE/DEAD sperm viability kit according to the manufacturer's instructions (Molecular Probes, Eugene, OR, USA). In all experiments, sperm were incubated in a water bath adjusted to $39^{\circ} \mathrm{C}$.

\section{Purification of PL glycoproteins}

The PL was isolated according to a procedure described by Sasanami et al. (2002). The PL was then dissolved in $1 \%$ SDS $(\mathrm{w} / \mathrm{v})$ buffered at $\mathrm{pH} 6.8$ with $70 \mathrm{mmol} / \mathrm{l}$ Tris- $\mathrm{HCl}$ overnight at room temperature. After centrifugation at $10000 \mathrm{~g}$ for $10 \mathrm{~min}$, the supernatants served as a PL lysate and the protein concentrations of the samples were measured using a BCA protein assay kit (Pierce, Rockford, IL, USA). The PL lysate was separated on one-dimensional SDS-PAGE, performed as described by Laemmli (1970) under non-reducing conditions on $12 \%(\mathrm{w} / \mathrm{v})$ polyacrylamide for separating gel. The samples (750 $\mu \mathrm{g}$ of protein per gel) were applied to $5 \%$ $(\mathrm{w} / \mathrm{v})$ stacking gel without comb for lane casting. After electrophoresis, the gel was stained with Copper Stain (Bio-Rad Laboratories), and $175 \mathrm{kDa}$ (dimeric ZP1), $97 \mathrm{kDa}$ (monomeric ZP1), and $35 \mathrm{kDa}$ (ZP3) bands were excised. The individual proteins were eluted by incubating the gel slices with $0.1 \%$ SDS (w/v) buffered at $\mathrm{pH} 8.0$ with $100 \mathrm{mmol} / \mathrm{ml}$ Tris- $\mathrm{HCl}$ overnight at $25^{\circ} \mathrm{C}$ with constant shaking. The eluent was then extensively dialyzed against water, lyophilized, and dissolved in $20 \mathrm{mmol} / \mathrm{ml}$ Tris- $\mathrm{HCl}(\mathrm{pH} \mathrm{8.0)}$. The protein concentrations of the samples were measured as described above.

\section{PNGase F digestion}

The PL lysate was digested with PNGase F, an amidase that cleaves between the innermost $\mathrm{N}$-acetylglucosamine and asparagines residues of $\mathrm{N}$-linked glycoproteins (E.C.3.5.1.52; New England Biolabs, Beverly, MA, USA), according to the manufacturer's instructions. Briefly, the PL lysate ( $1 \mathrm{mg}$ of protein) was mixed with $1 / 10$ volume of $10 \times \mathrm{G} 7$ buffer (0.5 M sodium phosphate, $\mathrm{pH} 7.5)$ and $10 \%(\mathrm{v} / \mathrm{v}) \mathrm{NP}-40$. The mixture was incubated in the presence or absence of $50000 \mathrm{U}$ of PNGase $\mathrm{F}$ at $37^{\circ} \mathrm{C}$ for $20 \mathrm{~h}$. It was then separated on one-dimensional SDSPAGE and the dimeric ZP1 or monomeric ZP1 was purified as described above. For the control experiments, (-PNGase $\mathrm{F}$ in panels $\mathrm{A}$ and $\mathrm{B}$ of Figs 1 and 2), the PL lysate was treated in the same manner except for the 

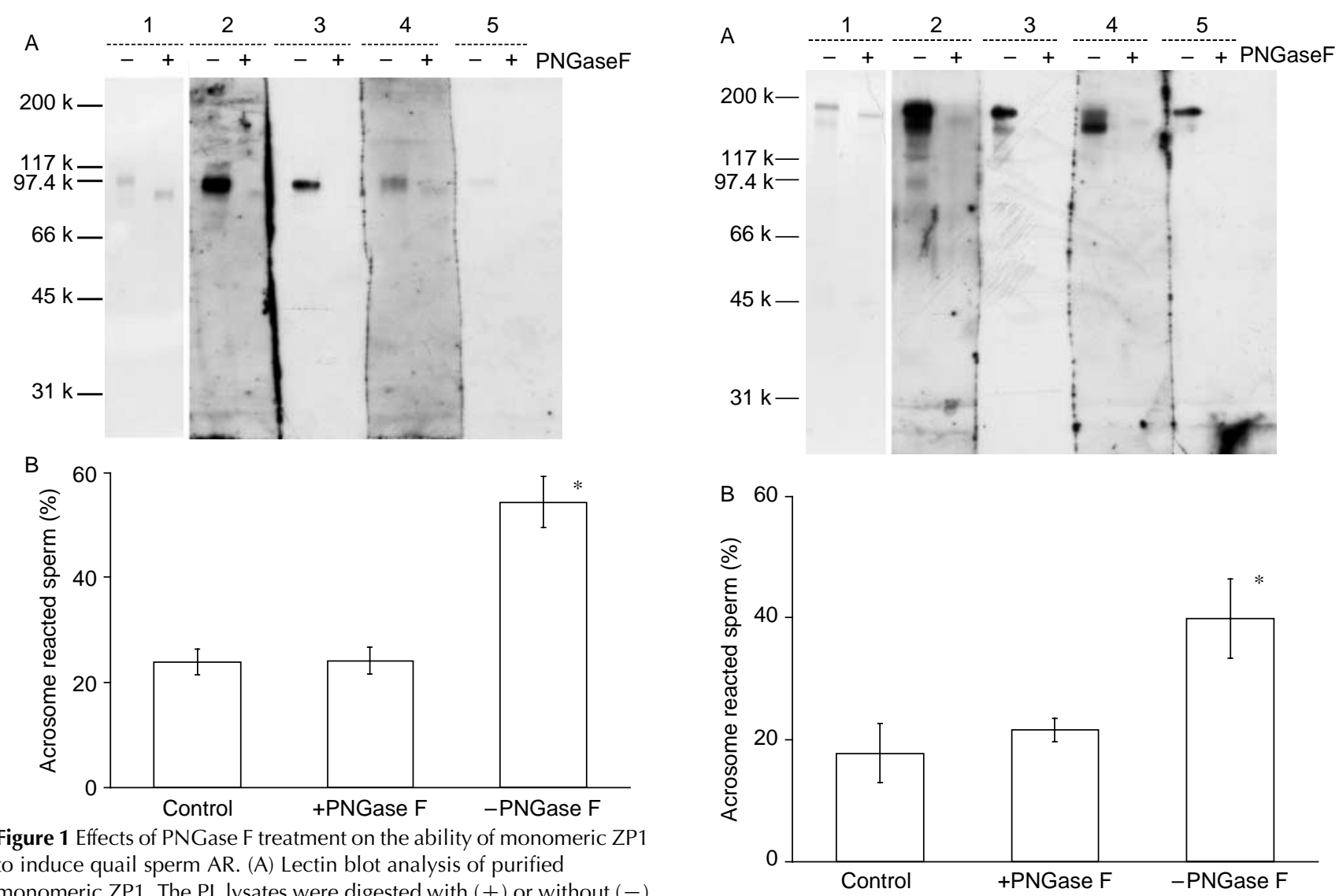

Figure 1 Effects of PNGase $F$ treatment on the ability of monomeric ZP1 to induce quail sperm AR. (A) Lectin blot analysis of purified monomeric ZP1. The PL lysates were digested with $(+)$ or without $(-)$ PNGase $\mathrm{F}$ as described in Materials and methods. They were then separated on SDS-PAGE (0.1 $\mu \mathrm{g}$ per lane), transblotted onto PVDF membranes, and incubated with $\mathrm{RCA}_{120}$ (panel 2, 1:10 000), PHA-E 4 (panel 3, 1:8000), WGA (panel 4, 1:2000) or LCA (panel 5, 1:5000). In panel 1, banding of the same sample stained with silver is shown. The panels of silver staining and lectin blot reflect one representative experiment out of a total of three independent experiments. (B) Ejaculated sperm were incubated for $10 \mathrm{~min}$ with vehicle alone (control), $1 \mu \mathrm{g} / \mathrm{ml}$ of the purified monomeric ZP1 digested with PNGase $\mathrm{F}(+\mathrm{PNGase} \mathrm{F})$, or $1 \mu \mathrm{g} / \mathrm{ml}$ of the purified monomeric ZP1 treated without PNGase F ( - PNGase F). The percentage of spermatozoa without acrosome was calculated. Values are shown as mean \pm s.D. of three independent experiments. Values with superscripts $\left({ }^{*} P<0.01\right)$ are significantly different from the control.

omission of PNGase F. The dimeric ZP1 or the monomeric ZP1 after the treatments was purified as described above.

\section{Assessment of acrosomal status}

Spermatozoa were diluted to a final concentration of $1 \times$ $10^{7}$ spermatozoa $\mathrm{ml}^{-1}$ in HBSS containing $0.1 \%(\mathrm{v} / \mathrm{v})$ of proctodeal gland secretion. The AR was induced by incubating the cells with $\mathrm{Ca}^{2+}$ ionophore A23187 dissolved in dimethylsulfoxide (DMSO; 0, 0.3, 1.0, or $3.0 \mu \mathrm{mol} / \mathrm{l}$; Sigma) for up to $30 \mathrm{~min}$. The reaction was stopped by incubating the samples with formaldehyde (final concentration of $3.7 \%(\mathrm{w} / \mathrm{v})$ ). The percentage of

Figure 2 Effects of PNGase F treatment on the ability of dimeric ZP1 to induce quail sperm AR. (A) Lectin blot analysis of purified dimeric ZP1. The PL lysates were digested with (+) or without (-) PNGase F as described in Materials and methods, and were detected with $\mathrm{RCA}_{120}$ (panel 2, 1:10 000), PHA-E 4 (panel 3, 1:8000), WGA (panel 4, 1:2000) or LCA (panel 5, 1:5000) as described in the legend for Fig. 1. In panel 1: banding of the same sample stained with silver. The panels of silver staining and lectin blot reflect one representative experiment out of a total of three independent experiments. (B) Ejaculated sperm were incubated for $10 \mathrm{~min}$ with vehicle alone (control), $1 \mu \mathrm{g} / \mathrm{ml}$ of the purified dimeric ZP1 digested with PNGase F (+PNGase F), or $1 \mu \mathrm{g} / \mathrm{ml}$ of the purified dimeric ZP1 treated without PNGase F (-PNGase F). The percentage of spermatozoa without acrosome was calculated. Values are shown as mean \pm s.D. of three independent experiments. Values with superscripts $\left({ }^{*} P<0.01\right)$ are significantly different from the control.

acrosome-reacted sperm was determined microscopically. Samples of the cells treated to induce the AR were smeared on poly-L-lysine coated microscope slides. After air drying, sperm smears were stained with $1 \mu \mathrm{g} / \mathrm{ml}$ propidium iodide (Sigma) for $10 \mathrm{~min}$. The slides were washed between incubations by dipping them in PBS for $5 \mathrm{~min}$. After washing, slides were mounted in glycerol and examined under a fluorescence microscope equipped with an interference-contrast apparatus with a $40 \times$ objective (BX 50, Olympus Optics, Tokyo, Japan) and photographed. At least five areas $0.237 \mathrm{~mm}^{2}$ were randomly selected for enumeration. The acrosome status 
was observed based on the presence (acrosome-intact sperm) or absence (acrosome-reacted sperm) of the acrosome, which is observed as a propidium iodidenegative structure located on the tip of the sperm nucleus. In order to investigate the effects of the purified $\mathrm{PL}$ glycoprotein on the AR, spermatozoa was incubated with or without dimeric, monomeric ZP1, or ZP3 for 10 min. After incubation, the acrosome status was observed using the same procedure as that used for A23187 treatment. To test the effects of pertussis toxin (PTX; Calbiochem, La Jolla, CA, USA), the membranepenetrating ADP-ribosyltransferase inhibitor of G protein, on the A23187- or PL glycoprotein-initiated $A R$, sperm were incubated with the test substance in the presence or absence of PTX $(2 \mu \mathrm{g} / \mathrm{ml})$ for $10 \mathrm{~min}$. Their acrosomal status was then observed. Appropriate solvent controls (DMSO, $20 \mathrm{mmol} / \mathrm{l}$ Tris- $\mathrm{HCl}\left(\mathrm{pH}\right.$ 8.0) or $\mathrm{H}_{2} \mathrm{O}$ for A23187, purified glycoproteins or PTX respectively) were utilized in parallel in all experiments and the final concentration of each solvent was adjusted to $0.1 \%(\mathrm{v} / \mathrm{v})$.

\section{Lectin blot analysis and gel staining}

For the lectin blot analysis, the purified ZP1 treated with or without PNGase $\mathrm{F}$ was separated on SDS-PAGE under non-reducing conditions $(0.1 \mu \mathrm{g}$ of protein per lane) and was transferred to PVDF membranes (Immobilon-P, Millipore, Bedford, MA, USA) (Matsudaira 1987). The membranes were incubated with three changes of saline buffered at $\mathrm{pH} 7.4$ with $10 \mathrm{mmol} / \mathrm{l}$ Tris- $\mathrm{HCl}$ containing $0.1 \%(\mathrm{v} / \mathrm{v})$ Tween $20(\mathrm{TBS}-\mathrm{T})$ for $10 \mathrm{~min}$ at room temperature in order to inhibit non-specific binding. The membrane was reacted with lectins, conjugated to horseradish peroxidase for $1 \mathrm{~h}$ at room temperature, and the reactive bands were detected as described previously (Pan et al. 2000). The lectins used were Ricinus communis agglutinin $\left(\mathrm{RCA}_{120}\right)$, which interacts with $\mathrm{N}$-acetylglucosamine-containing glycopeptides (Baenziger \& Fiete 1979), Phaseolus vulgaris agglutinin $\left(\mathrm{PHA}_{-} \mathrm{E}_{4}\right)$, which binds to $\mathrm{N}$-acetylglucosamine linked to $\beta 1,4$ to the $\beta$-linked mannose residue in the core (Cummings \& Kornfeld 1982), and Triticum vulgare agglutinin (WGA), specific for terminal $\mathrm{N}$-acetylglucosamine, sialic acids (Debray et al. 1981), and Lens culinaris agglutinin (LCA), which recognizes $\alpha 1,6$-fucosylated $\mathrm{N}$-glycans of a complex-type glycopeptide (Yamamoto et al. 1982) purchased from Honen Corp. (Tokyo, Japan).

To detect the protein of PL lysate or the purified glycoprotein, samples separated on SDS-PAGE under non-reducing conditions were detected with a silver staining kit (Wako Pure Chemicals, Tokyo, Japan).

\section{Statistical analysis}

All AR percentage data were transformed to the arcsine of their square root. The Duncan's multiple range test was used for a comparison of the group mean difference. Differences were considered statistically significant when $P<0.05$.

\section{Results}

\section{Analysis of AR of quail sperm}

In order to establish a quantitative assay for quail sperm $A R$, we observed sperm that had been incubated with or without A23187. As shown in Fig. 3A, sperm incubated with vehicle alone had a propidium iodide-negative structure on the tip of the nucleus (arrowhead). The size of this structure ranged from 2 to $4 \mu \mathrm{m}$ in length. In contrast, in the case of the spermatozoa treated with $3 \mu \mathrm{mol} / \mathrm{ml}$ of A23187, this structure was found to be missing (panel B). In order to validate this simple method for quail AR assay, we next incubated the sperm with various concentration of A23187 (Fig. 3C), and the percentage of sperm without acrosome was calculated. It was found that the percentage of sperm for which the acrosome had disappeared increased in a dose-dependent manner. In addition, a time-course study indicated that the acrosome-reacted
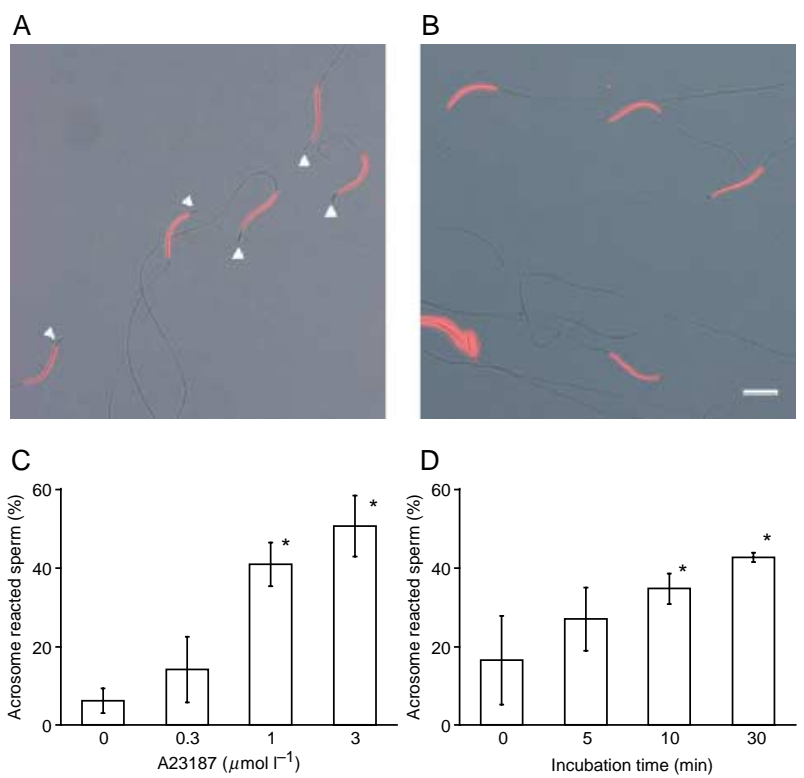

Figure 3 Effects of A23187 on quail sperm AR. (A and B) Ejaculated sperm were incubated with (panel B) or without (panel A) $3 \mu \mathrm{mol} / \mathrm{l}$ of A23187 for $10 \mathrm{~min}$ at $39^{\circ} \mathrm{C}$. Acrosomal status was evaluated based on the presence (acrosome-intact sperm, arrowhead in panel A) or absence (acrosome-reacted sperm, panel B) of their acrosome. Shown are representative results of repeated experiments. Bar $=10 \mu \mathrm{m}$. (C) Ejaculated sperm were incubated with $0,0.3,1.0$, or $3.0 \mu \mathrm{mol} / \mathrm{l}$ of A23187 for $10 \mathrm{~min}$, and the percentage of spermatozoa without acrosome was then calculated. Values were mean \pm s.D. of seven independent experiments. Values with superscripts $\left({ }^{*} P<0.01\right)$ are significantly different from the $0 \mu \mathrm{mol} / \mathrm{l}$ culture. (D) A time-course study of the induction of AR was performed. Sperm were incubated with $1.0 \mu \mathrm{mol} / \mathrm{l}$ of $\mathrm{A} 23187$ for $0,5,10$, or $30 \mathrm{~min}$. The percentage of spermatozoa without acrosome was calculated. Values are mean \pm s.D. of five independent experiments. Values with superscripts $\left({ }^{*} P<0.01\right)$ are significantly different from the 0-min culture. 
sperm increased in a time-related manner and that $10 \mathrm{~min}$ of incubation led the sperm to induce AR significantly in the presence of $1 \mu \mathrm{mol} / \mathrm{ml}$ of A23187 (Fig. 3D). These results indicated that the sperm $A R$ in quail could be simply analyzed by staining the sperm with a DNAstaining dye like propidium iodide.

\section{Sperm AR is induced by ZP1 stimulation in qauil}

In order to identify the proteins possessing activity to induce the $A R$, we purified two major glycoproteins, ZP3 and ZP1, from the PL lysate. As shown in Fig. 4A, rerun of the purified ZP3 (lane 2) as well as the dimeric (lane 3) or monomeric ZP1 (lane 4) produced a dominant band migrating at a molecular weight of 35,175 , and $97 \mathrm{kDa}$ respectively. These results suggest that each glycoprotein purified by means of the methods in this study was practically pure. To test the ability of the individual glycoprotein to induce the AR, we incubated the sperm with various concentrations of the purified glycoproteins. Treatment of spermatozoa with increasing doses of purified monomeric ZP1 for 10 min led to a concentration-dependent stimulation of the AR (Fig. 4C). The purified dimeric ZP1 had similar effects (Fig. 4D). On the other hand, the addition of purified ZP3 failed to induce the AR at any doses tested (Fig. 4B). Collectively, these results indicate that both monomeric and dimeric ZP1 possess activity to trigger the AR in Japanese quail.

\section{Effects of PTX on ZP1- or the A23187-induced AR}

In order to investigate the involvement of Gi protein in initiation of the AR, we evaluated whether the ZP1- or A23187-induced AR was affected by PTX, which is an inhibitor of the Gi protein function. As depicted in Fig. 5, incubation of the sperm with PTX completely inhibited the effects of ZP1 (Fig. 5A). It is not due to the toxic effect of PTX, because the motility and the viability of the sperm were not altered by the addition of PTX (data not shown). No significant difference was found between the percentage of acrosome-reacted sperm in the control and PTX culture. Similar results were obtained in the case of the A23187-induced AR, since the effects of A23187 on AR induction were significantly inhibited by the PTX treatment (Fig. 5B).

\section{Effects of PNGase F digestion on the ZP1-stimulated AR}

We next attempted to determine which region of the ZP1 molecule is involved in AR induction. We therefore investigated whether removal of $\mathrm{N}$-glycans attached to the ZP1 molecule affect the ability to induce the AR. When PL lysate was treated with PNGase F, the band of monomeric ZP1 was shifted to migrate at a molecular weight of $90 \mathrm{kDa}$ (Fig. 1A, panel 1, lane +). We next performed a lectin blot analysis of the proteins in order to confirm the absence of $\mathrm{N}$-linked oligosaccharides in ZP1 digested with PNGase F. As shown in the figure, the purified monomeric ZP1 without treatment reacted with

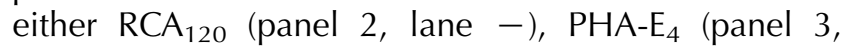
lane -), WGA (panel 4, lane -), or LCA (panel 5, lane -), which are known to recognize $N$-linked oligosaccharides, while the PNGase F digestion eliminated (panels 2, 3, and 5 lane + ) or markedly attenuated (panel 4 lane + ) the reactivity of ZP1 to the lectins used. These results suggested that our PNGase $\mathrm{F}$ treatment efficiently remove the $\mathrm{N}$-glycans from the ZP1, with only trace amounts of $\mathrm{N}$-glycans remaining on the ZP1 after PNGase $\mathrm{F}$ digestion. When the ejaculated sperm were incubated with ZP1, which were treated in the absence of PNGase F ( - PNGase F), the percentage of acrosomereacted sperm was significantly increased compared with that of the vehicle alone group (control). This result suggests that the course of treatments used for the PNGase $F$ digestion itself did not result in an inactivation of ZP1 activity for the stimulation of AR. When the cells were incubated with N-glycan-deficient ZP1 (+PNGase $\mathrm{F})$, the percentage of the acrosome-reacted sperm was significantly decreased compared with that in the - PNGase F group, and reached the levels equivalent to those in the control group. As shown in Fig. 2, similar results were obtained in the case of the experiments using purified dimeric ZP1. These results demonstrated that the $N$-linked oligosaccharides attached to both monomeric and dimeric ZP1s play an important role in triggering the AR in quail sperm.

\section{Discussion}

Our previous study demonstrated that the holes that are a result of hydrolysis of the PL are observed when PL obtained from preovulatory follicles is incubated with ejaculated sperm in vitro (Kuroki \& Mori 1997). This result implied the presence of $A R$ inducer in the PL of quail oocyte. Horrocks et al. (2000) previously developed a method to detect the AR of chicken spermatozoa based on the differential binding of FITC labeled PNA (FITC-PNA) to acrosome-intact but not acrosome-reacted spermatozoa. However, our preliminary experiments using their method indicated that the FITC-PNA does not bind to quail sperm irrespective of the treatment of A23187 (data not shown). In order to investigate the AR of quail sperm, we established a method for discriminating acrosome-reacted from acrosome-intact sperm. Since the acrosome in quail sperm is large enough to be visualized under a light microscope (Korn et al. 2000), we employed a simple method in which the sperm nucleus was stained with fluorescence DNA dye. As expected, we successfully differentiated between acrosome-reacted and acrosome-intact sperm based on the presence or absence of the acrosome (Fig. $3 \mathrm{~A}$ and B). 
By means of this method, the results in the present study clearly demonstrate for the first time that both monomeric and dimeric ZP1, one of the major constituents of the PL,
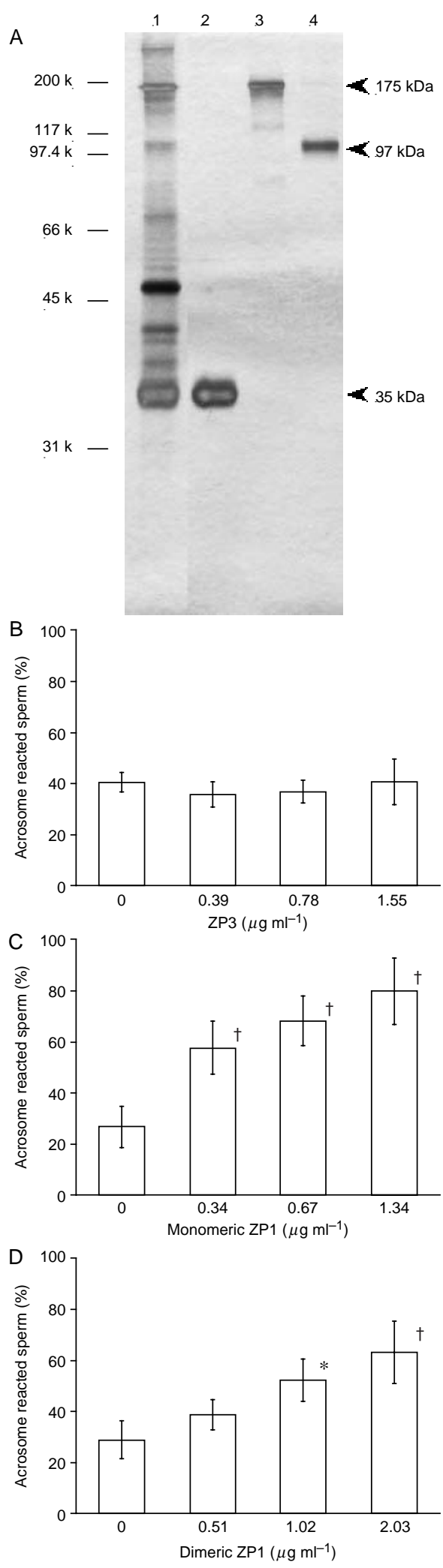

possess activity for stimulation of the AR in quail. In accordance with our findings, another recent study has demonstrated that dimeric ZP1 stimulates sperm AR in the chicken (Okumura et al. 2004). However, the results of this previous study also suggest that monomeric ZP1 has no effect on induction of the AR. They speculate that the inefficacy of monomeric ZP1 for AR induction was due to its secondary structure suggested by $C D$ spectra measurement, i.e. the monomeric ZP1 is rich in unordered structure, whereas the dimeric one was estimated to have ordered secondary structure, which might contribute to the activity for AR induction. Although the reason for this discrepancy between chicken and quail is not investigated in this study, these authors used a gel filtration technique with buffer containing $6 \mathrm{~mol} / \mathrm{l}$ urea, whereas we employed SDSPAGE under non-reducing conditions for the purpose of ZP1 purification. On the other hand, Bausek et al. (2004) have demonstrated that both ZP3 purified from a culture supernatant of granulosa cells and ZP1 obtained from the serum of laying hens are bound to the acrosomal region of rooster sperm by means of immunofluorescent microscopy. In addition, they also showed that both ZP3 and ZP1 interact with a $180 \mathrm{kDa}$ protein present in the lysate of rooster sperm. Since they did not analyze AR induction in the chicken, it is unknown why the acrosome still remained on the head of the rooster sperm after incubation with ZP1, an AR inducer in both chicken and quail. However, it should be noted that we purified ZP1 from the PL of the largest preovulatory follicle, just prior to fertilization, while they used ZP1 of serum origin.

It is quite interesting that sperm binding to the $\mathrm{PL}$ in chicken appears to be mediated by both ZP1 and ZP3 (Bausek et al. 2004). Although both ZP3 and ZP1 bind to the acrosomal region of rooster sperm, only ZP1 possesses the activity for AR induction (Okumura et al. 2004 and in this study). These results implied the following two possibilities: (1) there are multiple ligands on the sperm surface, and one of which is responsible for the specific binding to ZP3, while the other one contributes to ZP1 binding and AR induction or (2) ZP3 and ZP1 share a common ligand with different

Figure 4 Effects of purified PL glycoproteins on the induction of $A R$ in Japanese quail. (A) The purified ZP3, (lane 2, $0.1 \mu \mathrm{g}$ per lane), the purified dimeric ZP1 (lane 3, $0.1 \mu \mathrm{g}$ per lane), or the purified monomeric ZP1 (lane 4, $0.1 \mu \mathrm{g}$ per lane) were separated on SDS-PAGE under non-reducing conditions, and were detected with silver staining. The PL lysate ( $1 \mu \mathrm{g}$ per lane) was also separated on SDS-PAGE under non-reducing conditions (lanes 1 ) as a reference. Shown are representative results of repeated experiments. (B, C and D) Ejaculated sperm were incubated with ZP3 $(0,0.39,0.78$, or $1.55 \mu \mathrm{g} / \mathrm{ml}$, panel A), monomeric $(0,0.34,0.67$, or $1.34 \mu \mathrm{g} / \mathrm{ml}$, panel B) or dimeric ZP1 $(0$, $0.51,1.02$, or $2.03 \mu \mathrm{g} / \mathrm{ml}$, panel C) for $10 \mathrm{~min}$. The percentage of spermatozoa without acrosome was calculated. Values of each panel are shown as mean \pm s.D. of four independent experiments. Values with superscripts $\left({ }^{*} P<0.05,{ }^{+} P<0.01\right.$ respectively) are significantly different from the respective control culture $(0 \mu \mathrm{g} / \mathrm{ml})$. 

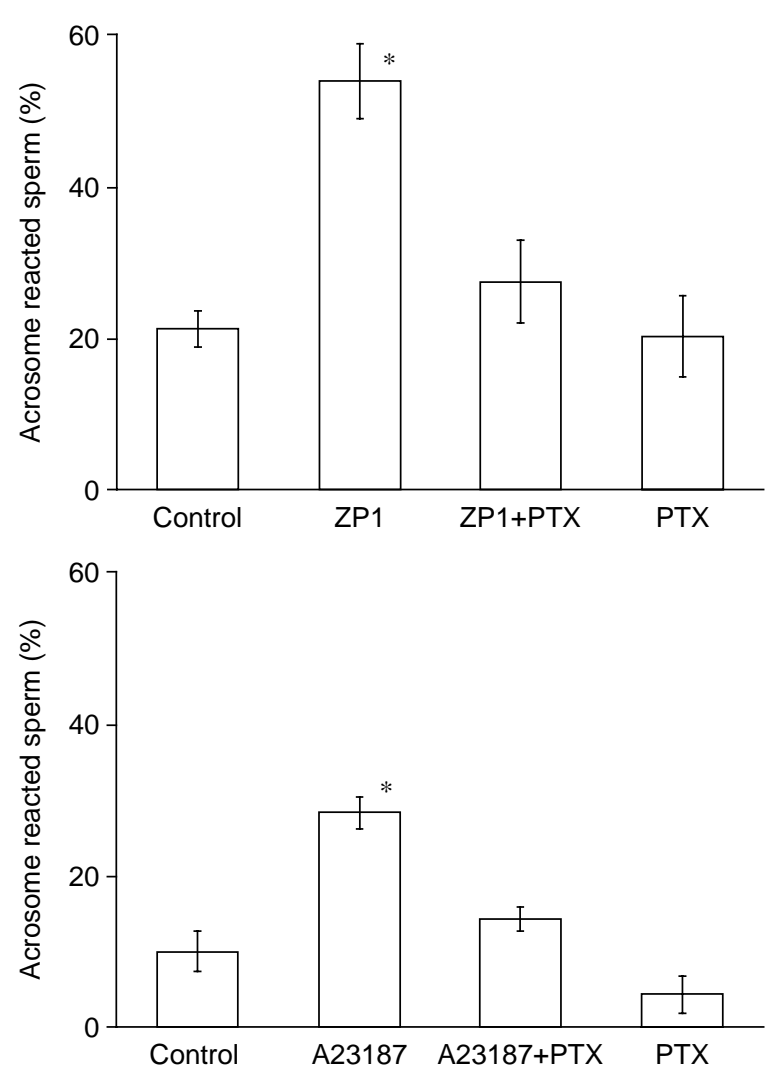

Figure 5 Effects of pertussis toxin on ZP1 - or A23187-initiated quail sperm AR. (A) Ejaculated sperm were incubated with vehicle alone (control), $1 \mu \mathrm{g} / \mathrm{ml}$ of purified ZP1 alone (ZP1), ZP1 with $2 \mu \mathrm{g} / \mathrm{ml}$ pertussis toxin $(\mathrm{ZP1}+\mathrm{PTX})$, or pertussis toxin alone (PTX) for $10 \mathrm{~min}$. The percentage of spermatozoa without acrosome was calculated. Values are shown as mean \pm s.D. of four independent experiments. Values with superscripts $(* P<0.01)$ are significantly different from the control. (B) Ejaculated sperm were incubated with vehicle alone (control), $10 \mu \mathrm{mol} / \mathrm{l}$ of $\mathrm{A} 23187$ alone (A23187), A23187 with $2 \mu \mathrm{g} / \mathrm{ml}$ pertussis toxin (A23187+PTX), or pertusis toxin alone (PTX) for $10 \mathrm{~min}$. The percentage of spermatozoa without acrosome was calculated. Values are shown as mean \pm s.D. of three independent experiments. Values with superscripts $\left({ }^{*} P<0.01\right)$ are significantly different from the control.

affinity, and that the signal transduction that mediates the $A R$ in quail spermatozoa does not proceed after binding with ZP3. Although these are only hypotheses, efforts are presently underway to identify the specific sperm ligand(s) for egg envelope glycoproteins, ZP3, and ZP1.

The role of $\mathrm{PL} \mathrm{N}$-glycans in sperm-egg interactions have been demonstrated in chicken because $\mathrm{N}$-glycans released from the PL by PNGase F as well as the materials bound to WGA-conjugated agarose can induce the AR (Horrocks et al. 2000). Moreover, the addition of galactose to terminal $\mathrm{N}$-acetylglucosamine residues of PNGase F-released $\mathrm{N}$-glycans by galactosyl transferase suppresses the AR-inducing capacity of the oligosaccharide preparation, suggesting the involvement of $\mathrm{N}$-linked glycans with a terminal $\mathrm{N}$-acetyl-D-glucosamine residue on AR induction (Horrocks et al. 2000). However, the identification of which carrier of $\mathrm{N}$-glycans could be involved in AR induction remained to be accomplished. In our results, the removal of $\mathrm{N}$-glycans from ZP1, which was confirmed by the absence of the reactivity to lectins, had no effect on induction of the AR (Figs $1 \mathrm{~B}$ and 2B), demonstrating for the first time the important role of $\mathrm{N}$-glycans of both monomeric and dimeric ZP1 in the induction of $A R$ in Japanese quail. Although it appears that there is participation by $\mathrm{N}$-linked oligosaccharides, we did not investigate the involvement of $o$-glycans of ZP1 in the induction of the AR in quail. However, Robertson et al. (2000) have demonstrated that the pretreatment of chicken PL with o-glycanase does not reduce the number of holes after incubation with ejaculated spermatozoa in vitro. A determination of the carbohydrate structure of ZP1 required for AR induction in quail must await future analysis, however, our lectin blot analysis indicated the presence of $\mathrm{N}$-acetylglucosamine in the sugar moiety of ZP1 protein, which is recognized with $\mathrm{RCA}_{120}$ and $\mathrm{PHA}-\mathrm{E}_{4}$. It is of interest to note that the sperm hydrolysis of the PL in chicken was inhibited when $\mathrm{N}$-acetylglucosamine was included in the incubation mixture (Robertson et al. 2000).

A common element of the ZP-initiated AR mechanism in eutherian mammals is involvement of the Gi protein, and PTX, which is an inhibitor of Gi protein function, is known to inhibit the ZP-initiated AR in mammalian sperm (Florman \& Ducibella 2006). Interestingly, it has been reported that PTX does not inhibit the progesterone-induced AR of human and mouse sperm (Tesarik et al. 1993, Murase \& Roldan 1996). In addition, PTX also does not inhibit the acetylcholine-stimulated AR in the mouse, suggesting the involvement of a PTXinsensitive receptor like the nicotinic acetylcholine receptor in AR induction (Son \& Meizel 2003). These data also suggest that different physiological stimuli may utilize different signal transduction pathways to induce the sperm AR. Our results demonstrate that both ZP1and A23187-induced AR are significantly inhibited when PTX is included in the incubation mixture. These results also indicate that the ZP1 or A23187 might be acting through a Gi protein-mediated mechanism similar to that in the zona-initiated AR in mammalian sperm.

A number of important issues regarding quail fertilization remain to be addressed. Specifically, it is not yet known how acrosome-reacted sperm could remain on the surface of the PL and then penetrate the membrane. In an analogous situation, it has been suggested that the adhesion between the acrosome-reacted sperm and the ZP may be mediated by the binding sites on the sperm inner acrosomal membrane that interact with ZP2 in several mammalian species (Bleil et al. 1988, Mortillo \& Wassarman 1991, Tsubamoto et al. 1999). It remains to be resolved whether or not similar ZP2-mediated sperm retention on the PL could function in the case of the quail fertilization. It should be noted that our recent observation using electron microscopy has demonstrated that calcium-coated sperm-associated bodies $(\mathrm{SAB})$ were 
accompanied with the hole in the PL of quail fertile egg, and suggested that the SAB assist fertile spermatozoa in binding to the $\mathrm{PL}$, making holes in the membrane and passing through it (Rabbani et al. 2006). Since the sperm bind with the SAB via the posterior portion of the flagella, it appears possible that the $S A B$ could control sperm motility and might facilitate PL penetration by amplifying the forward thrust from the flagellum in quail. In support of this idea, it has been suggested that sperm penetration of the eutherian ZP is achieved by lysis of the egg coat by acrosomal enzymes in conjunction with forward thrust from the sperm-tail oscillation (Bedford 1998).

In conclusion, our results indicate that $\mathrm{N}$-linked glycans on ZP1 play an essential role in triggering the AR in Japanese quail. Additional studies will be needed to identify the machinery mediating the specific binding of spermatozoa with the PL as well as the organization of the three-dimensional structure of the $\mathrm{PL}$, including the targeting of ZP1 and ZP3 to the PL from the bloodstream, and from the granulosa cells respectively.

\section{Acknowledgements}

We would like to thank Professor Norio Yoshizaki, Faculty of Agriculture, Gifu University, for his invaluable advice and discussions regarding the experimental design. We thank Miss Mie Higuchi and Ayaka Yamazaki for their technical assistance. This work was supported in part by Grant-in-Aids for Scientific Research (18780210 to T S) from the Ministry of Education, Culture, Sports, Science and Technology, Japan. The authors declare that there is no conflict of interest that would prejudice the impartiality of this scientific work.

\section{References}

Ashizawa K, Wishart GJ, Katayama S, Takano D, Ranasinghe ARAH, Narumi K \& Tsuzuki Y 2006 Regulation of acrosome reaction of fowl spermatozoa: evidence for the involvement of protein kinase $C$ and protein phosphatase-type 1 and/or- type 2A. Reproduction 131 1017-1024.

Baenziger JU \& Fiete D 1979 Structural determinants of Ricinus communis agglutinin and toxin specificity for oligosaccharides. Journal of Biological Chemistry 254 9795-9799.

Bausek N, Waclawek M, Schneider WJ \& Wohrab F 2000 The major chicken egg envelope protein ZP1 is different from ZPB and is synthesized in the liver. Journal of Biological Chemistry 275 28866-28872.

Bausek N, Ruckenbauer HH, Pfeifer S, Schneider WJ \& Wohlrab F 2004 Interaction of sperm with purified native chicken ZP1 and ZPC proteins. Biology of Reproduction 71 684-690.

Bedford JM 1998 Mammalian fertilization misread? Sperm penetration of the eutherian zona pellucida is unlikely to be a lytic event. Biology of Reproduction 59 1275-1287.

Bleil JD, Greve JM \& Wassarman PM 1988 Identification of a secondary sperm receptor in the mouse egg zona pellucida: role in maintenance of binding of acrosome-reacted sperm to eggs. Developmental Biology 128 376-385.

Cummings RD \& Kornfeld S 1982 Characterization of the structural determinants required for the high affinity interaction of asparagineslinked oligosaccharides with immobilized Phaseolus vulgaris leukoagglutinating and erythroagglutinating lectins. Journal of Biological Chemistry 257 11230-11234.
Debray H, Decout D, Stecker G, Spik G \& Montreuil J 1981 Specificity of twelve lectins towards oligosaccharides and glycoproteins related to N-glycosylations. European Journal of Biochemistry 117 41-55.

Florman HM \& Ducibella T 2006 In Physiology of Reproduction, 3 edn, Vol 1, pp 55-112. Ed. JD Neill. St Louis, MO, USA: Elsevier Academic Press.

Gilbert AB, Evans AJ, Perry MM \& Davidson MH 1977 A method for separating the granulosa cells, the basal lamina and the theca of the preovulatory ovarian follicle of the domestic fowl (Gallus domesticus). Journal of Reproduction and Fertility 50 179-181.

Harris JD, Hibler DW, Fontenot GK, Hsu KT, Yurewicz WC \& Sacco AG 1994 Cloning and characterization of zona pellucida genes and cDNAs from a variety of mammalian species: the ZPA, ZPB and ZPC gene families. DNA Sequence 4 361-393.

Horrocks AJ, Stewart S, Jackson L \& Wishart GJ 2000 Induction of acrosomal exocytosis in chicken spermatozoa by inner perivitellinederived N-linked glycans. Biochemical and Biophysical Research Communications 278 84-89.

Howarth B 1990 Avian sperm-egg interaction: perivitelline layer possesses receptor activity for spermatozoa. Poultry Science 69 1012-1015.

Korn N, Thurston RJ, Pooser BP \& Scott TR 2000 Ultrastructure of spermatozoa from Japanese quail. Poultry Science 79 86-93.

Koyanagi F, Masuda S \& Nishiyama H 1988 Acrosome reaction of cock spermatozoa incubated with the perivitelline layer if the hen's ovum. Poultry Science 67 1770-1774.

Kuroki M \& Mori M 1997 Binding of spermatozoa to the perivitelline layer in the presence of a protease inhibitor. Poultry Science $\mathbf{7 6} 748-752$.

Laemmli UK 1970 Cleavage of structural proteins during the assembly of the head of bacteriophage T4. Nature 227 680-685.

Lefievre L, Conner SJ, Salpekar A, Olufowobi O, Ashton P, Pavlovic B, Lenton W, Afnan M, IBrewis IA, Monk M, et al. 2004 Four zona pellucida glycoproteins are expressed in the human. Human Reproduction 19 1580-1586.

Matsudaira P 1987 Sequence from picomole quantities of proteins electroblotted onto polyvinylidene difluoride membranes. Journal of Biological Chemistry 262 10035-10038.

Mortillo S \& Wassarman PM 1991 Differential binding of gold-labeled zona pellucida glycoproteins $\mathrm{mZP} 2$ and mZP3 to mouse sperm membrane compartments. Development 113 141-149.

Murase T \& Roldan ER 1996 Progesterone and zona pellucida activate different transducing pathways in the sequence of events leading to diacylglycerol generation during mouse sperm acrosomal exocytosis. Biochemical Journal 320 1017-1023.

Okumura H, Kohno Y, Iwata Y, Mori H, Aoki N, Sato C, Kitajima K, Nadano D \& Matsuda T 2004 A newly identified zona pellucida glycoprotein, ZPD and dimeric ZP1 of chicken egg envelope are involved in sperm activation on sperm-egg interaction. Biochemical Journal 384 191-199.

Pan J, Sasanami T, Nakajima S, Kido S, Doi Y \& Mori M 2000 Characterization of progressive changes in ZPC of the vitelline membrane of quail oocyte following oviductal transport. Molecular Reproduction and Development 55 175-181.

Pan J, Sasanami T, Kono Y, Matsuda T \& Mori M 2001 Effects of testosterone on production of perivitelline membrane glycoprotein ZPC by granulosa cells of Japanese quail (Coturnix japonica). Biology of Reproduction 64 310-316.

Rabbani MG, Sasanami T, Mori M \& Yoshizaki N 2006 Sperm-egg interaction is mediated by a sperm-associated body in quail. Development, Growth and Differentiation 48 33-40.

Robertson L, Wishart GJ \& Horrocks AJ 2000 Identification of perivitelline $\mathrm{N}$-linked glycans as mediators of sperm-egg interaction. Journal of Reproduction and Fertility 120 397-403.

Sasanami T, Pan J, Doi Y, Hisada M, Kohsaka T, Toriyama M \& Mori M 2002 Secretion of egg envelope protein ZPC after C-terminal proteolytic processing in quail granulosa cells. European Journal of Biochemistry 269 2223-2231. 
Sasanami T, Pan J \& Mori M 2003 Expression of perivitelline membrane glycoprotein ZP1 in the liver of Japanese quail (Coturnix japonica) after in vivo treatment with diethylstilbestrol. Journal of Steroid Biochemistry and Molecular Biology 84 109-116.

Smith J, Paton IR, Hughes DC \& Burt DW 2005 Isolation and mapping the chicken zona pellucida genes: an insight into the evolution of orthologous genes in different species. Molecular Reproduction and Development 70 133-145.

Son JH \& Meizel S 2003 Evidence suggesting that the mouse sperm acrosome reaction initiated by the zona pellucida involves an $\alpha 7$ nicotinic acetylcholine receptor. Biology of Reproduction $\mathbf{6 8}$ 1348-1353.

Spargo SC \& Hope RM 2003 Evolution and nomenclature of zona pellucida gene family. Biology of Reproduction 68 358-362.

Tesarik J, Carreras A \& Mendoza C 1993 Differential sensitivity of progesterone- and zona pellucida-induced acrosome reaction to pertussis toxin. Molecular Reproduction and Development 34 183-189.

Tsubamoto H, Hasegawa A, Nakata Y, Naito S, Yamasaki N \& Koyama K 1999 Expression of recombinant human zona pellucida protein 2 and its binding capacity to spermatozoa. Biology of Reproduction 61 1649-1654.
Waclawek M, Foisner R, Nimpf J \& Schneider WJ 1998 The chicken homologue of zona pellucida protein-3 is synthesized by granulosa cells. Biology of Reproduction 59 1230-1239.

Wassarman PM 1999 Mammalian fertilization: molecular aspect of gamete adhesion, exocytosis, and fusion. Cell 96 175-183.

Win MM, Tatemoto H, Ashizawa K \& Nakada T 2006 Effects of diethylstilbestrol administration on sperm penetration into the inner perivitelline layer of Japanese quail. Journal of Poultry Science $\mathbf{4 3}$ 67-74.

Wyburn GM, Aitken RNC \& Johnston HS 1965 The ultrastructure of the zona radiata of the ovarian follicle of the domestic fowl. Journal of Anatomy 99 469-484.

Yamamoto K, Tsuji T \& Osawa T 1982 Requirement of the core structure of a complex-type glycopeptide for the binding to immobilized lentil-and pea-lectins. Carbohydrate Research 110 283-289.

Received 14 July 2006

First decision 7 August 2006

Revised manuscript received 11 August 2006

Accepted 8 September 2006 